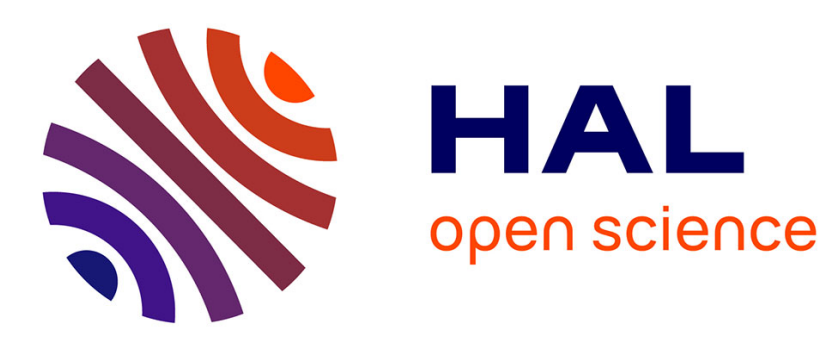

\title{
High Order Nonlinearities and Mixed Behavior in Micromechanical Resonators
}

\author{
Najib Kacem, Sebastien Hentz, Sébastien Baguet, Régis Dufour
}

\section{To cite this version:}

Najib Kacem, Sebastien Hentz, Sébastien Baguet, Régis Dufour. High Order Nonlinearities and Mixed Behavior in Micromechanical Resonators. 10th biennial International Conference on Vibration Problems, Sep 2011, Prague, Czech Republic. hal-00633113

\section{HAL Id: hal-00633113 https://hal.science/hal-00633113}

Submitted on 15 Nov 2019

HAL is a multi-disciplinary open access archive for the deposit and dissemination of scientific research documents, whether they are published or not. The documents may come from teaching and research institutions in France or abroad, or from public or private research centers.
L'archive ouverte pluridisciplinaire HAL, est destinée au dépôt et à la diffusion de documents scientifiques de niveau recherche, publiés ou non, émanant des établissements d'enseignement et de recherche français ou étrangers, des laboratoires publics ou privés. 


\title{
High Order Nonlinearities and Mixed Behavior in Micromechanical Resonators
}

\author{
N. Kacem, S. Hentz, S. Baguet and R. Dufour
}

\begin{abstract}
This paper investigates the sensitivity of the third order nonlinearity cancellation to the mixed (hardening and softening) behavior in electrostatically actuated micromechanical resonators under primary resonance at large amplitudes compared to the gap. We demonstrate the dominance of the mixed behavior due to the quintic nonlinearities, beyond the critical amplitude when the third order mechanical and electrostatic nonlinearities are balanced. We also report the experimental observation of a strange attraction which can lead to a chaotic resonator.
\end{abstract}

Key words: Nonlinear dynamics, mixed behavior, strange attraction

\section{Introduction}

The large potential of microelectromechanical systems (MEMS) and nanoelectromechanical systems (NEMS) has been widely demonstrated for ultrasensitive force and mass sensing applications [1, 2]. However, it is a challenge to enhance their dynamic range (DR) by achieving large amplitudes comparable to the gap in the case of electrostatically driven resonators without altering their frequency stability. Combined with the noise mixing issue [3] when the resonator is driven in the nonlinear regime, this leads to a drastic limitation on the sensor resolution.

N. Kacem

Université de Lyon, CNRS INSA-Lyon, LaMCoS UMR525, F-69621 Villeurbanne France, e-mail: najib.kacem@insa-lyon.fr

S. Hentz

CEA/Leti - MINATEC, Microsystems Components Laboratory, F-38054 Grenoble France

S. Baguet

Université de Lyon, CNRS INSA-Lyon, LaMCoS UMR525, F-69621 Villeurbanne France

R. Dufour

Université de Lyon, CNRS INSA-Lyon, LaMCoS UMR525, F-69621 Villeurbanne France 
An alternative to overcome this physical limitation is the hysteresis suppression by nonlinearity cancellation which has been the aim of extensive research work lately and requests the study of nonlinear dynamics of MEMS and NEMS. Most nonlinear models dealing with the nonlinearity cancellation in capacitive micro and nanoresonators includes nonlinear terms up to the third order $[4,5]$. So even experimentally observed [5], the nonlinearity cancellation is limited by the high order nonlinearities which occur proportionally sooner with respect to the resonator size. Precisely, the onset of the mixed behavior is close to the critical amplitude for resonant NEMS sensors.

Indeed, accurate nonlinear models [6, 7] for large amplitudes includes all main sources of nonlinearities without Taylor series expansion of the nonlinear electrostatic forces. Kacem et al. developed complete multiphysics models including high order nonlinear terms and demonstrated analytically the ability to cancel out the nonlinearities in MEMS and NEMS capacitive clamped-clamped beam resonators [6] as well as cantilevers [8]. Moreover, Kacem and Hentz [9] identified experimentally the mixed behavior predicted in [6] i.e. they demonstrated the physical significance of the quintic nonlinear terms.

In this paper, we report experimentally the high sensitivity of the third order nonlinearity cancellation to the mixed behavior on a MEMS resonator under primary resonance at large amplitudes with respect to the gap. Based on a high order nonlinear model, the fabricated resonator was designed with a large ratio between its width and the sensing gap in order to maximize its global critical amplitude. We demonstrate the ability to reach very large amplitudes of order $90 \%$ of the sensing gap without pull-in occurrence. Moreover, strange attraction on a mixed behavior is experimentally identified which can lead in some configurations to chaos.

\section{Experimental investigations}

The fabricated resonator shown in Figure 1 consists in a silicon doubly clamped beam electrostatically in plane actuated and detected using two electrodes (electrode 1 for actuation and 2 for sensing) which allows for 2 port electric measurements.

It has been fabricated on $200 \mathrm{~mm}$ SOI (Silicon on insulator) wafers and microelectronic silicon processes. It is $200 \mu \mathrm{m}$ long, $2 \mu \mathrm{m}$ thick, $5 \mu \mathrm{m}$ wide, the actuation gap is $1 \mu \mathrm{m}$, and the detection gap $300 \mathrm{~nm}$. It has been designed to enable the compensation of the nonlinearities thanks to a compact analytical model [6] including all main sources of nonlinearities (electrostatic and mechanical) presenting non linear terms up to the seventh order as shown in Equation (1).

$$
\begin{array}{r}
\left(1+\sum_{i=1}^{4} \mu_{i} a_{1}^{i}\right)\left(\ddot{a}_{1}+c \dot{a}_{1}\right)+\sum_{i=0}^{2} \zeta_{i} a_{1}^{i} \cos (\Omega t) \\
+\sum_{i=0}^{7} \chi_{i} a_{1}^{i}+\sum_{i=3}^{5} \zeta_{i} a_{1}^{i-3} \cos (2 \Omega t)=0
\end{array}
$$




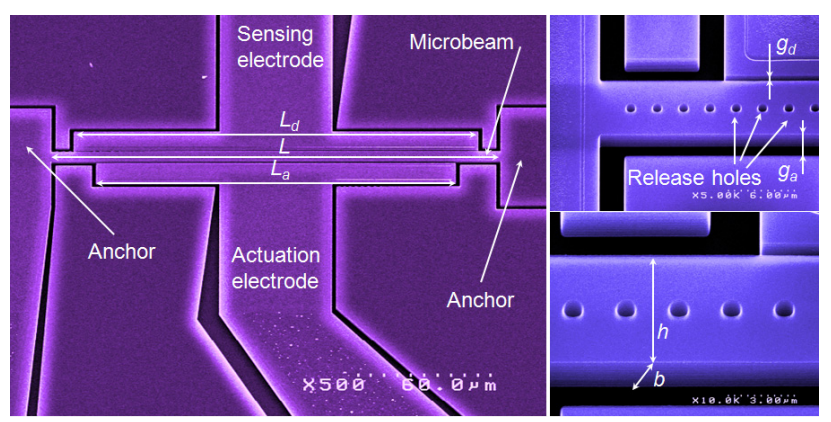

Fig. 1 SEM images of the fabricated resonator designed using a complete nonlinear model [7].

The device was placed in a vacuum chamber (down to $1 \mathrm{mTorr}$ ), and the 2-port electrical measurements were performed at room temperature using a low noise lock-in amplifier (Signal Recovery 7280).

As a first step, the critical behavior is approached in order to slowly access the nonlinear regime. Figure 2 displays a hardening behavior for which the mechanical nonlinearities are much higher than the electrostatic nonlinearities. Indeed, for a $D C$ voltage of $1 V$, the nonlinear negative Duffing nonlinearity is very low which implies a high quality factor $\left(Q \approx 10^{4}\right)$. Hence, in this configuration, the mechanical critical amplitude is smaller than the electrostatic one $\left(A_{c} \approx 80 \mathrm{~nm}\right)$. The hardening resonance peak of Figure 2 has been obtained in sweep up and down frequency which displays a critical behavior at an output voltage of $7 \mu \mathrm{V}$. The fabricated device

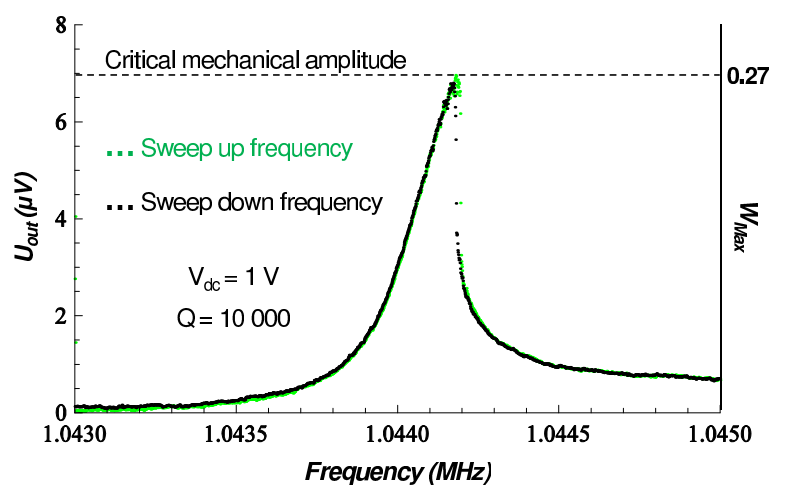

Fig. 2 Measured critical hardening resonance peak for $V_{a c}=0.2 \mathrm{~V} . W_{\operatorname{Max}}$ is the displacement of the beam at its middle point normalized by the gap $g_{d}$.

has the characteristic of large width to gap ratio and consequently can potentially display a linear compensated behavior when both electrostatic and mechanical nonlinearities are balanced by increasing the nonlinear spring softening effect. 
In order to approach this operating point (hysteresis suppression), the $D C$ voltage has been increased to $3 \mathrm{~V}$ and $5 \mathrm{~V}$ as shown in Figure 3 where the resonance curves have been electrically characterized in sweep up and down frequency.

Since the negative nonlinear stiffness is proportional to the square of $V_{d c}$ (for $V_{d c}>>V_{a c}$ ), unlike the first peak obtained at a $D C$ voltage of $3 V$, the second one (left curve) measured for $V_{d c}=5 \mathrm{~V}$ displays nonlinear branches in the softening domain characterized by a complete modification of the dynamic bifurcation topology. The frequency shift between both curves due to the negative stiffness is about $2 \mathrm{KHz}$.

Moreover, the first peak measured at $V_{d c}=3 V$ displays a quality factor $Q=6000$ which has been measured on a linear curve at a low $A C$ voltage and the same $D C$ voltage. The resonance curve, in this case, is strangely nonlinear (hardening behavior). The third bifurcation is the highest one in the hardening domain obtained in sweep up frequency where the corresponding curve displays two regimes: a first fast in amplitude variation and a second slow with a slope approaching zero. The first bifurcation is obtained in sweep down frequency and intercepts the sweep up frequency curve in a small part of the slow regime and the entire fast regime of amplitude variation. The strange nonlinear hardening behavior obtained experimentally can be explained by a strong dynamic perturbation due to the increase of the softening nonlinearitie.

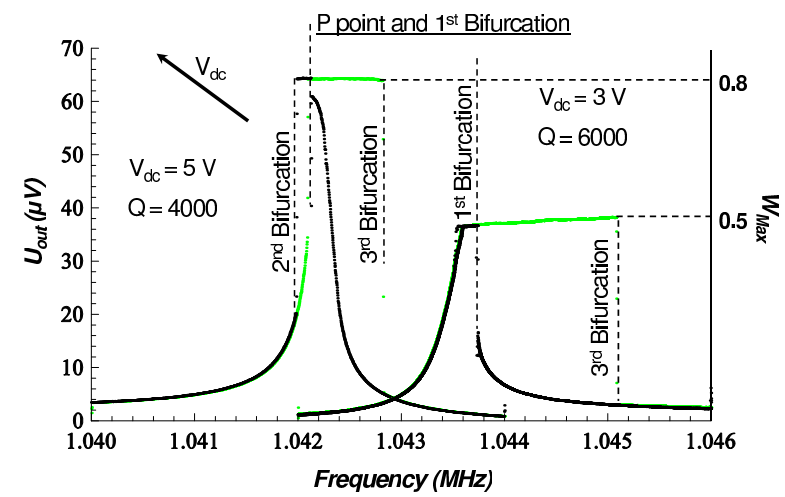

Fig. 3 Measured strange hardening and mixed resonance peaks for $V_{a c}=0.2 \mathrm{~V}$ showing the changes on the bifurcation topologies. $P$ is the mixed behavior initiation point and the third bifurcation is the highest point in the hardening domain.

In Figure 3, the left resonance curve measured at $V_{d c}=5 \mathrm{~V}$ displays a quality factor $Q=4000$. Using the analytical model of a 2 ports nonlinear resonator developped in [6], for these parameters, the resonator should display a linear resonance peak obtained by the compensation of the mechanical and the electrostatic nonlinearities. Unlike the mixed behavior (hardening-softening) demonstrated in [9], the experimental peak measured at $V_{d c}=5 \mathrm{~V}$ displays clearly a mixed behavior starting by a softening branch and ending by a hardening one where the peak amplitude is around three times the critical amplitude displayed in Figure 2. 
Particularly, in this mixed behavior, the $P$ point and the first bifurcation have the same frequency and the hardening domain is reduced in comparison with the first resonance curve of Figure 3. Actually, for the fabricated resonator, the fifth order nonlinear terms are no more negligible when it is used to operate close to the hysteresis suppression point. Indeed, the compensation of the nonlinearities is sensitive to the highly unstable mixed behavior.

Once the mixed behavior was reached, we continue the increase of the $D C$ voltage in order to track the transition from a mixed to a softening behavior as shown in Figure 4. The first peak has been measured at a $D C$ voltage of $7.5 \mathrm{~V}$ where the branches obtained in sweep down frequency enable the capture of two bifurcation points: the first one is the $P$ point which corresponds to the initiation of the mixed behavior domain and the second one is the highest bifurcation point in the hardening domain.

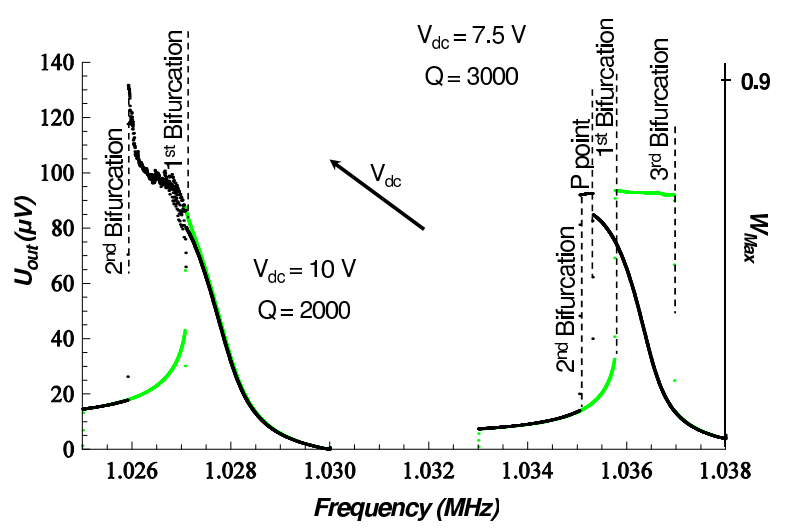

Fig. 4 Measured mixed and softening resonance peaks for $V_{a c}=0.2 \mathrm{~V}$.

Then, in sweep up frequency, the output voltage $V_{\text {out }}$ follows the curve characterized by the bifurcation points 1 and 3 . When the frequency of the first bifurcation point is reached, a strange attractor brings the resonator oscillation to the upper branch in the hardening domain instead of following the sweep down frequency curve (jump to the downer branch) which should be physically easier where the basins of attraction are quite larger. This is another illustration of the physical importance of the quintic nonlinearities. Hence, it is difficult to achieve large amplitudes for MEMS and NEMS capacitive resonators without altering their frequency stability.

In order to suppress the mixed behavior, the effect of the hardening nonlinearities is reduced in the second peak of Figure 4 for $V_{d c}=10 \mathrm{~V}$ and an estimated quality factor $Q=2000$. The electrostatic nonlinearities are amplified with respect to the mechanical nonlinearities which brings the third bifurcation point at the same frequency as the first one. Remarkably, the resonator has reached very large oscillations up to $90 \%$ of the sensing gap without pull-in occurence. Nevertheless, the highest 
branch in the softening domain displays an inflection point (potentially due to the high order hardening nonlinearities) followed by a dynamic relaxation at the jump down bifurcation point.

\section{Conclusions}

In summary, we have demonstrated the high sensitivity of the third order nonlinearity cancellation to the mixed behavior in nonlinear capacitive clamped-clamped MEMS resonators under primary resonance at large oscillations. The experimental investigations were performed on a fabricated resonator designed using a complete analytical nonlinear model in order to balance the third order nonlinearities and drive the resonator linearly at high amplitudes. Beyond the critical amplitude, the quintic nonlinearities drastically limit the operating domain of the nonlinearity cancellation. Moreover, strange attractions become possible which can lead to undesirable chaotic sensors. Consequently, for nonlinear optimized resonant MEMS designs, the onset of the mixed behavior must be considered.

Acknowledgements The authors gratefully acknowledge financial support from the CEA LETI and I@L Carnot institutes (NEMS Project) and from the MNTEurop Project.

\section{References}

1. Rugar, D., Budakian, R., Mamin, H.J., and Chui, B.W.: Single spin detection by magnetic resonance force microscopy, Nature, 430, 2004, pp. 329.

2. Jensen, K., Kim, K., and Zettl, A.: An atomic-resolution nanomechanical mass sensor, Nature Nanotechnology, 3, 2008, pp. 533.

3. Kaajakari, V., Koskinen, J.K., and Mattila, T.: Phase noise in capacitively coupled micromechanical oscillators, IEEE Trans. Ultrason. Ferroelectr. Freq. Control, 52, 2005, pp. 2322.

4. Kozinsky, I., Postma, H.W.Ch., Bargatin, I., and Roukes, M.L: Tuning nonlinearity, dynamic range, and frequency of nanomechanical resonators, Appl. Phys. Lett., 88, 2006, pp. 253101.

5. Shao, L.C., Palaniapan, M., and Tan, W.W.: The nonlinearity cancellation phenomenon in micromechanical resonators, J. Micromech. Microeng., 18, 2008, pp. 065014.

6. Kacem, N., Hentz, S., Pinto, D., Reig, B., and Nguyen, V.: Nonlinear dynamics of nanomechanical beam resonators: improving the performance of NEMS-based sensors, Nanotechnology, 20, 2009, pp. 275501.

7. Kacem, N., Baguet, S., Hentz, S., Dufour, R.: Computational and quasi-analytical models for non-linear vibrations of resonant MEMS and NEMS sensors, International Journal of NonLinear Mechanics, 46, 2011, pp. 532.

8. Kacem, N., Arcamone, J., Perez-Murano, F., and Hentz S.: Dynamic range enhancement of nonlinear nanomechanical resonant cantilevers for highly sensitive NEMS gas/mass sensor applications, J. Micromech. Microeng., 20, 2010, pp. 045023.

9. Kacem, N., and Hentz S.: Bifurcation topology tuning of a mixed behavior in nonlinear micromechanical resonators, Appl. Phys. Lett., 95, 2009, pp. 183104. 